\title{
Physical Education as a means of health and working efficiency improvement of population
}

\author{
Rezeda R. Khasanova ${ }^{1 . a}$, Tatyana V. Saricheva ${ }^{1}$, Antonina A. Soboleva ${ }^{1}$, Vladimir I. Andreev ${ }^{1}$, Oleg I.Zagrevsky $^{2}$, Darya \\ U. Nikonova ${ }^{1}$, Liudmila V.Smagliy ${ }^{1,3, a}$. \\ ${ }^{1}$ Tomsk Polytechnic University, 634050 Lenin Avenue, 30, Tomsk, Russia \\ ${ }^{2}$ Tomsk State University, 634050 Lenin Avenue, 36, Tomsk, Russia \\ ${ }^{3}$ Siberian State Medical University, 634050, Moskovskiy trakt 2, Tomsk, Russia
}

\begin{abstract}
In this article historical and contemporary influence of physical education (PE) and sport instances are shown in economic indices. The notion "health", as well as the influence of physical education and sport at its level, is given. Health-detrimental behavior (malnutrition, physical inactivity, alcohol abuse and smoking) can be the cause of chronic diseases and have a significant influence on health of citizens. Experimental data, which approve that bad working conditions and health-detrimental behavior can discourage productivity and extend the periods of temporary incapacity for work, are marked. In addition, there is an access to the majority of the adult population, which provides a perfect opportunity for healthy lifestyle campaign. Consequently, the campaigns aimed at health improvement of the employable population at their working places, have potentials for a wide population segment engagement, which cannot be accessible for other medical campaigns. Experimental data on increase in labour productivity and economic effectiveness improvement by means of physical education are given; the latter was applied to people at their work during the soviet period, when the large-scale research was made. The necessity of physical education integration in the productive process is proved, which will finally have a positive impact on the whole economy.
\end{abstract}

\section{Introduction}

Economists distinguish three main types of economic resources: natural, human and material values. Among the enumerated economic resources, human ones are characterized by the fact that they can be regarded as economy development resources, consuming material values and service activities at the same time. In addition, among human resources there are the so-called "labour forces" which are a narrower form of a human resources expression and include not only employable population at active working age but also working teenagers and pensioners. Regular exercises and training are necessary tools for good health, which decrease the risks of getting chronic diseases and psychological disorders such as diabetes, heart diseases, depressions and infectious diseases. [1-3]. The remarkable thing is that over the last two decades there has increased the number of chronic diseases in their hard form. This circumstance led to putting emphasis on living standards research and estimating relationships between the latter and the health level.

In current economic conditions, there is a profound reconstruction of the economic strategy, the key points for which cause qualitative changes to occur not only in material production, but also in non-production sphere's sectors. In the field of PE and sport, qualitative changes occur principally in its new role in economic reproduction. PE serves as an intensification factor of the employable part of population, providing a healthy lifestyle: professional longevity extension, assistance in well-minded spending of after-hours, healthy human needs development. The growth of material and financial expenses, additional labour resources involvement, the $\mathrm{PE}$ and sport significancy increase in social reproduction actualize the issues of the given service industries effectiveness in conditions of developed market system. In such a way, the new stage of economic development and an economic and social situation decline predetermine the necessity of detailed research of human resources, especially the employable population aiming at prognostics of possible economic consequences.

A human resources qualitative characteristic is described by some basic criteria: human capital (the ability to bring income is estimated), human potential, work-force, labour potential (labour resources in qualitative measurement). Such components as health, morality, proactive attitude, professionalism refer not

\footnotetext{
a Corresponding author: lud.smagly@yandex.ru, hasanova_rezeda@mail.ru
} 
only to a person, but also to different economic systems [4-6]. It is undoubted that health is the main criterion which best characterizes working capacity of the population and is the most significant. Poor health condition of a worker influences his/her capacity for taking part in community service and also defines the absolute value and the structure of society expenses for work-force. It is proved that the profit from human capital is much more than from physical. In this connection, investments in health service, education, PE, sport and science are justified. Thus it is apparent that health is a basic criterion which characterizes the work potential of citizens, and other components depend upon it.

In addition to the above-mentioned, it is discovered by researchers that regional economic conditions have a significant influence on health of the society, which is demonstrated by index numbers of the birth rate, general mortality, mortality of the employable part of population and general morbidity. Medical-demographic situation disregard can lead to big expenses and can create deflationary socioeconomic problems which require more resources in comparison to those in case of their prevention.

At the bottom of PE effectiveness there are socioeconomic factors of social development. The connection of Physical Education and sport with Economics does not mean that the function of this sector is fully covered by production operation, but it underlines the unity and interconnection of economic and social factors of social reproduction. Thus the indemnity of work-force expenses is a significant component in economic development, and, at the same time, it is a complex social and biological process. In view of this, there appears the necessity to mention two aspects of effectiveness - social and economic.

Beginning with the nineties of the $20^{\text {th }}$ century and till nowadays, health deterioration and length of life shortening of the population are observed everywhere in Russia, leading to the decrease of such economic resource as human potential of the country. According to statistical data, the medical-demographic decline shows itself in changing the age pattern of the population there is a decrease in employable part of the population, on the other hand, there is an increase in treatment expenses. A low level of staff training expenses payoff is observed because of length of life shortening, investments in disease prevention become more expensive. A health indicator is the main criterion characterizing the labour potential of the population and other components depending upon it.

\section{Results}

\subsection{Relationship of health and economic results}

The effectiveness of PE and sport application has an effect on the following components: health status (20\%), morbidity and length of life $(15 \%)$; influence over the after-hours spending structure (10\%); influence over equation of social and economic life conditions of citizens and villagers (5\%), influence over the healthy lifestyle development $(40 \%)$, the level of physical development of an average voluntary worker $(10 \%)$. The described components are connected to the employability of the population, it means they have a significant influence on the social-economic effect at the place of production by means of refinement of work. Consequently, PE and sport perform some social functions which are predominantly aimed at healthy lifestyle cultivation; it means they are very important for economics of every country. These are the main reasons why governmental activity is aimed at sport and PE development control by means of promulgation of relevant regulations, governmental campaigns and investments. Besides, Sport and Physical culture by means of promotion of health and general development of a man play an important role in economy of the country $[5,6]$. Studying the economic aspect of PE and sport has obtained a particular actuality, because for a modern society it is very important to know not only the sum spent on PE, but also the benefits from these expenses. It is necessary to differentiate finance and economic effectiveness of $\mathrm{PE}$ and sport. Economic effectiveness of PE and sport reflects the results of the impact on efficiency of labor, cost saving. Economic effectiveness of PE and sport is estimated with the help of the following key figures: aggregate consumption growth of material and non-material values; social labour productivity growth as a result of PE and sport impact on working capacity; GDP growth as a result of $\mathrm{PE}$ and sport impact and growth of population engaged in sports activities. The aspect of economic effectiveness of PE and sport has a great practical value for defining development perspectives of service industries and a rate of its growth in market conditions.

Health is the main value of life, it stands on the highest level in the hierarchy of human needs, because it is essential for a successful social and economic development. In opinion of many leading Russian scientists, the earlier given definition of "health" is not that concrete. For example, A.G. Schedrina offers a more detailed characterization of the term "health": "Health is an integral, multidimensional, dynamic condition (including its positive and negative data), which develops in the process of its genetic potential realization in conditions of a particular social and ecological environment, allowing a person to perform his/her biological and social functions to different extents." In our opinion, this definition discovers the connection between health and economic result to the full extent. [1].

In their representation of the term "health", national scientists put emphasis on integrative data of a person, underlining that human health is predominantly a process of saving and development of its mental and physical qualities, optimized working capacity and community relief in case of maximal length of life.

Investigations showed that daily dynamics of human working capacity is defined by periods of physiological processes to a large extent, being under the influence of exogenous (connected with environmental changes) and endogenous (rhythm of heartbeats, breath, blood 
pressure, mental and physical activity, deep sleep or not) factors.

High working capacity in every activity category, as a rule, appears only in that case if vital rhythm is harmonized with biological rhythms of this or that organism and its psychophysiological functions. Thus, the more precise the coincidence of the beginning of working activity with the rise in tonus of vital functions of an organism is, the more efficiently tasks are performed; tolerance of an organism increases, fatigability decreases and state of health is getting better. According to generalized data of special investigations, it was found out that working capacity of people who exercise regularly increases in $0,6-10 \%$.

According to the statement of Russian physiologist V. Vvedensky: "People usually get tired not because of working too much but because they do not do a good job", meaning that their work organization leaves to be desired. These basic principles and conditions of productivity, which were formulated by him, should be taken into consideration. Joining a regular training is connected with creating good attitude to PE, understanding its social role and personal necessity to be involved in a particular system of knowledge in order to lead a healthy lifestyle.

Exercising evolves adaptive morphological and functional reconstructions of an organism that finds its reflection in a gain in health and has a medicinal effect in many cases.

In addition to the above-mentioned, proper work and rest organization is an important condition for preservation of health, good working capacity and successful acquirement of shop-floor skills which means that regular exercising has a positive influence on economy.

\subsection{Physical education influence on economic effectiveness}

A significant interest in the influence of PE on human health level and increase in labour productivity refers to the beginning of the 20th century. In twenties and thirties, this field of knowledge generated strong interest of USSR scientists, as the industrialization was the main task not only from an economic point of view but also from a political one. [2, 3].

The importance of exercising, aiming at prophylaxis of work-related diseases and increase in working capacity, was underlined in the letter of Commissariat of the USSR A. Semashko, sent in January, 1930, to regions. This letter served as a kind of impact on activation of investigations aimed at defining the meaning of PE in prophylaxis and treatment of work-related diseases. [9].

In Western Siberia these investigations were carried out in a Territorial scientific research cabinet of medical control, created in 1929 in Novosibirsk. The working sphere of the cabinet was to investigate occupational gymnastics, as a means of increase in working capacity and a gain in health of workers. In order to make it more efficient they specified the forms of its organization, conducted experiments of exercising influence during a working day in companies and factories. [10, 11]. This experience was shared with specialists in PE of quickly developing Kuzbass and builders of the first Siberian metallurgical complex in Stalinsk. There were organized PE brigades of builders, and in Kemerovo a newly opened pit was named "sportive", because there were a lot of such brigades. Working capacity of pitmensportsmen was exemplary for all workers of Kuzbass and pride for the whole Siberian region, which was marked at celebration of PE and sport, held in Novosibirsk in 1931 [12].

The investigation of the experience of gymnastics organization in conditions of coal-pit as one of the factors of the working capacity increase was very important because the production programme enlargement for this segment of production encouraged economic growth not only of the region but the country at large [12]. In 1933, pits of Kuzbass were assigned for conducting active works of $\mathrm{PE}$ inoculation in the process of production of pitmen. Finally a very valuable material and diagrams of investigations were obtained [12].

Investigations of $\mathrm{PE}$ influence were carried out everywhere and in March 1933 there was an all Union Conference regarding scientific issues of $\mathrm{PE}$ and production and so on [12].

Investigations in this sphere continued in the $1950 \mathrm{~s}-$ 1960s. According to data of scientific research of that time, sportsmen's working capacity was averagely higher by $3.8-4.5 \%$, those who were on sick leave thrice less often. Sportsmen had better health as well. For example, people, working at several factories in Altaisky region became healthier by $20-30 \%$ due to active health promotion (factories of Transport Engineering, Engineering Tools, Biysk Chemical industrial complex, "Zarya", Barnaul Boiler-plant factory). The sportsmen of the bearing plant in Tomsk in the 1960 s were on sick leave thrice less often. In this regard, a maximal promotion of $\mathrm{PE}$ had an obvious economic profit. [13, 21].

At the beginning of the 1970s, a considerable investigation in the sphere of economic effectiveness of PE was carried out. High economic effectiveness of PE was clearly seen by the example of Novokuznetsk aluminum smelter of Kemerovo Oblast (1972). The sports complex "Alumintshik" had more than 4400 sportsmen, who made up $63.5 \%$ of all workers of the aluminum smelter. There were Spartakiada Games of health every year in 8 types of sport, in which every second worker took part. The administration of the smelter plant invested great funds to sports events, which were easily compensated. It was calculated, for example, that workers-sportsmen were often on sick leave thrice less than those who were not exercising, working capacity increased by $8-10 \%$ [14].

In 1981 in Tomsk a decision on the basis of sports complex of Oblast Sports Committee was made to organize a scientific health-promoting association "Health Center". Its tasks were prophylaxis of heart diseases among workers, reduction of working hours' loss because of sick leaves and working capacity increase. It was Tomsk scientific center AMS USSR and "Kontur" association initiative. A scientific health- 
promoting association held a wide range of sports activities, propagandized a healthy lifestyle and medical knowledge, aimed at prophylaxis of heart diseases among workers of Tomsk companies. Among workers of the "Kontur" association and other industrial companies of Tomsk, pertaining to the prophylaxy groups of healthy people, were formed. They took part in sports and medical events with the help of the sport complex "Tom". All sports events were paid by the "Kontur" association, but the profit was amazing. Only in 1984 sick list expenses were reduced by 35 thousand of rubles. Meanwhile regardless this propaganda in Tomsk oblast, in the middle of 1980s there were high losses of working time because of sick leaves, 15000 workers were daily absent, and 28 million rubles were spent on sick lists every year. The high level of morbidity was observed among geologists, builders and workers of timber industry, oil and gas industry and agriculture. The main problem with the administrations of these companies was the low level of involvement of workers in sports events and there was also lack of facilities, staff and inventory. Only in 1984, 1 billion of working days were lost because of this, which equals to 4 million daily absent workers. In opinion of the government, health deterioration of the population was apparently connected to a lack of large-scale participation in PE and sport: in 1985, two-thirds of citizens of the country were not involved in PE events. [15].

In the nineties of the $20^{\text {th }}$ century in Russia, there was insufficient involvement of children and teenagers in PE and sport that in its turn had a negative impact on the health of young generation. In fact there was a complete absence of sports events, which was the cause of growth in alcoholism and drug dependency among children and teenagers which had a negative influence on social and economic numbers [20].

The presented analysis of historical facts proves one more time that PE and sports events have a positive influence on the economics of the country. In relation to this, a concept of long-term social and economic development of the Russian Federation till 2020 year was worked out and integrated. According to this concept, the role of PE and sport in development of human potential in Russia is one of the Key factors of demographic stabilization and decrease in mortality of the employable part of population [21].

According to statistical data, nowadays Russia significantly gets behind (nearly $11 \%$ of the employable part of the population are involved in regular PE and sports events) in comparison to the numbers of mature economies $(40-50 \%$ of the employable part of the population). One more fact that proves applicability of this issue is the average life span in Russia, which is on the $143 \mathrm{~d}$ place in the world (at the same level as in the poorest countries of Africa and Asia), taking into consideration the life expectancy numbers (67 years). Then there arises a question: how does the interconnection between PE and sport appear, length of life and economic numbers appear? The investigations prove that the connection of these three notions is obvious, i.e. those people who regularly go in for sport 1.5 times more rarely suffer from fatigue, 2 times more rarely - from diseases of the digestive system, 2.5 times more rarely - from high blood pressure, and 2 times more rarely - from infections and viruses. All in all, people who often go in for sport are 2 times more rarely absent from work because of health problems and they work harder than those who do not [21-23]. The described data can be explained by the fact that the loss of working hours of those going in for sports is 4.5 times less than those who ignore sports events. It was calculated in one of the investigations that every invested ruble in the PE sphere made 14 - 29 rubles profit, which was because of the reduction of the loss of working hours, an injury rate and treatment expenses. In addition, the increase of a relative proportion of workers going in for sports up to $50 \%$ will raise the economic effectiveness on an average by $35 \%$ [24].

During our investigation, one more question arose: what are the reasons of economic development of the country? According to a classical canon of economic theory, there are two ways of economic growth: extensive and intensive. In the basis of the extensive one, there is the enlargement of scale of production that can be reached by the amount of involved in production factors of production on the same technical basis: amount of workers' growth, increase in investments, increase in raw material volume. The intensive type of economic growth includes the integration in the process of production of new effective means, technology and processes, consequently, the economic growth can be reached because of factors of production improvement: earning power, regime of economy, scientifictechnological progress, workplace and production management improvement. It is obvious, that the key role in economy is played by labour resources, the productivity of which depends on the following factors: intellectual capabilities, medical condition, and length of life, at the heart of which there are regular exercises. It is scientifically proved that physical activities elongate active working age and favorably influence mental activity. In modern conditions of ageing of the population and a decrease in the birth rate, the influence of these factors has a very important meaning for the maintenance of economic growth rate. In this way, a worker who regularly goes in for sports, have less health problems, quickly kicks into gear and creates good environment around him/her, s/he is more self-motivated than others and consequently the companies, which involve their workers in sports events, are increasing their competitiveness of work force, independent of the economic sphere, and the rate of economic growth. PE and sport play a great role in the solution of socioeconomic problems', as they are a specific social and cultural sphere, which has a positive influence on important indices of economic growth of the country. The colossal economic and social effect because of PE and sports programs realization is proven. In this aspect going in for sport is a very important element in the process of reproduction of a qualitative work force and in labor force development. Nowadays, in conditions of socio-economic improvements, in modern Russia the issues of gaining in health are of a great importance, which has a positive influence on the economy of the country. For this reason, on the one hand, PE and sport 
development are paid a lot of attention and, on the other hand, this development is of high-priority in socioeconomic government policy.

Besides, according to Goskomstat data, nowadays in our country natural population decline is still stable $(0.7$ million a year), which proves again a bad demographic situation and health condition of the population.

\section{Conclusions}

It is apparent that the development of sport sphere results in the development by creating new working places and additional sources of national budget. Moreover, earlier we have already paid attention to that fact that PE and sport are key factors when it comes to promotion of health. Still the problem of involvement of population in regular sport activities is not solved. In order to solve this problem many companies spend a lot of money, aiming at attraction of the population to PE and sport. Such companies as "Gazprom", "Transneft" and others pay a lot of attention and spend a lot of money on PE and sport development, regularly organizing sport festivals for their workers, winners of which are awarded prizes. Many gyms with all available facilities are opened and so on. All enumerated events are aimed not only at the maintenance of the health level, but also at creation of a corporate spirit. Thus, the contemporary policy of these and some other companies is predominantly aimed at creation of economical incentive, which allows improving living standards and health of workers, having a positive impact on working efference of a company and on the whole country.

We should pay attention to the fact that economic indices of PE and sport effectiveness mentioned here allow with full confidence speaking on cost effectiveness of expenses for PE and sport development, mentioning the fact that they are not overhead but are good investments. At the same time, the described data demonstrate the necessity of a further dynamic development of PE and sport both from social and economic points of view. Thus, PE and sport become not only a sufficient component of a healthy lifestyle, but also a significant factor of economic growth, which can have a positive impact on all the spheres of expansion of production. On the ground of the stated facts, it is obvious that management system integration and clear milestones not only influence the economy of the country, but also make a sufficient contribution to global problems solution. All enumerated moments underline the fact that the contemporary society pays a lot of attention to qualitative improvement of working capacity including such tools as PE and sport development. From the point of view of the government, creating conditions for PE and sport development is an investment in human capital, in improvement of living standards of the population and finally in the increase of working efficiency which have a positive impact on the economy of the country. Thus, the technological progress in the sphere of production is viable in health and prosperity maintenance. During sports events aimed at promotion of health apart from technological progress we have to pay attention to public awareness campaign, explaining the information on health and its advantages.

\section{References}

1. R.M. Eime, J.T. Harvey, M.J. Charity, M.M. Casey, J.G.Z. van Uffelen, W.R. Payne. BMC Public Health. 15. 806 (2015)

2. R.R. Khasanova, O.I. Urazova, V.V. Novitsky. 4th International Interdisciplinary Conference on "Modern problems in systemic regulation of physiological functions”. 671-674 (2015)

3. M. Casey, W. Payne, R. Eime. Manag Leis.; 14. 112-124 (2009)

4. R.M. Mayberry, D.A. Nicewander, H. Qin, D. J. Ballard. Proc (Bayl Univ Med Cent). 19(2). 103-118 (2006)

5. P.A. Titov. Vestnik MGTU. 13 (1). 215-217 (2010)

6. V.K. Doev. Business economics: theory and practice. 9. 54-58 (2012)

7. S. Wu, D. Cohen, Y. Shi, M. Pearson, R. Sturm. Am J Prev Med. 40(2). 149-158 (2011).

8. E.B. Kahn, L.T. Ramsey, R.C. Brownson, G.W. Heath, E.H. Howze, K.E. Powell, E.J. Stone, M.W. Rajab, P. Corso. Am J Prev Med. 22(4 Suppl). 73107 (2002).

9. J.-H. Kim, E.-C. Park. BMC Public Health. 15. 783 (2015)

10. A.G. Schedrina. Medicine and education in Siberia. 5. 7 (2009).

11. T.V. Sarycheva Bulletin of Siberian medicine. 14 (3). 68-73 (2015)

12. Historical archive of Omsk Oblast. F. 28. L. 1. F. 117.

13. National archive of Novosibirsk oblast. F. P-906. L. 1. F. 4.

14. National archive of Novosibirsk oblast. F. 1810. Оп. 1. F. 24.

15. National archive of Novosibirsk oblast. F. P-906. Оп. 1. F. 4a.

16. National archive of Altay Territory. F. Р-1475. Оп 1. F. 166.

17. National archive of Kemerovo Oblast F. 75. Оп. 12. F. 87.

18. Contemporary history documentation center. F. 607. L. 13. F. 47.

19. M.S. Malashenko Culture. Spirituality. Society. 6. 161-170 (2013)

20. A.T. Prokopieva Theory and practice aspects of contemporary science. 8-4. 130-134 (2015)

21. A.A. Udina Pedagogical education and science. 3. 35-39 (2013)

22. A.V. Litvinov. Problems of modern economy. 3. 414-417 (2009)

23. A. Soboleva, R. Khasanova, V. Andreev, T. Sarycheva, L. Smagly. 8th World Conference on Educational Sciences (WCES-2016) : abstract book. 325 (2016)

24. STRATEGY of PE development in the Russian Federation till 2020 year. 
URL:http://www.minsport.gov.ru/documents/orders/ $\underline{1830 /}$ 